\title{
Does Upper Trapezius Activity Increase Substantially Over a 2-Minute Push-Up Bout?
}

\author{
Jennifer K Hewit*, Daniel A Jaffe and Alexander J Bedard \\ Department of Physical Education, United States Military Academy, USA
}

*Corresponding author: Jennifer Hewit, Department of Physical Education, United States Military Academy, 727 Brewerton Rd, West Point, NY 10996, USA

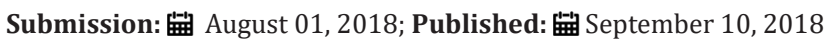

\begin{abstract}
Purpose: The aim of this study was to identify changes in the magnitude of muscle activity in the upper trapezius muscle group during a 2-minute push-up test.

Methods: Twenty-six college-aged males performed a 2-minute standardized push-up test. Upper Trapezius (UT) muscle activity was recorded throughout the test using surface electromyographic (EMG) electrodes. Individual portable force plates placed under each hand recorded the percent of body weight as a representation of force output over the hands throughout the bout.

Results: Normalized muscle activity increased $(p=0.23$ ) from the start to the end of the bout, while force output decreased significantly ( $p=0.00$ ) throughout the bout.

Conclusion: The decrease in force output through the hands does not appear to coincide with increased activity in the upper trapezius. It is speculated that the decrease in force output at the end of the bout is a result of the lower body supporting a larger percent of body weight (likely observed through increased hip flexion); an altered body position that requires less activation of the UT.
\end{abstract}

Keywords: Body weight; Force output; Muscle activity; Technique

\section{Introduction}

Push-ups are a muscular endurance exercise commonly performed by people of all different abilities and ages. Being able to control one's own body weight through a specific range of motion, as is done during the push-up, requires not only strength, but also awareness of body alignment and compensational strategies that might develop with the onset of fatigue. Being able to control the body and maintain the optimal positioning is crucial to mastering the exercise and, more importantly minimizing the potential for injury. The primary focus of literature that addresses the pushup is centered around the ideal hand position to either decrease elbow joint stresses or to maximize muscle activity in the pectorals and triceps [1-7]. However, over recent years there has been a shift in training modalities to create unstable supporting surfaces or increase the explosiveness of the task to further challenge the traditional body weight exercises. For example, push-ups are often performed with the hands or feet on a stability ball $[4,8,9]$ or suspension rings $[10,11]$ as opposed to on the ground. Similarly, many training programs will incorporate plyometric push-up variations to improve maximum force and power $[12,13]$. While this does create a more challenging training environment, it is important that a foundation of total body strength, control, and technique are achieved prior to moving to the unstable or explosive variations.
Activation of the primary (e.g. pectoralis major and triceps brachii) and secondary (e.g. anterior deltoid) muscles for the push-up are often of interest to researchers $[2,6,13,14]$. However, less attention has been given to the upper trapezius (UT) muscle when performing the push-up. Excessive activation of the UT compared to that of the Serratus Anterior may result in impingement syndrome and decreased effectiveness of the natural scapulothoracic rhythm [15]. Over-activation of the upper trapezius can typically be visually observed as elevation of the scapula and a shoulder shrugging motion. Optimal performance of the push-up includes neutral position of the shoulder or slightly depressed [16]. However, once an individual begins to tire, compensational movement patterns begin to develop. While these altered body positions and altered muscle activation patterns allow the movement to continue, the risk on incurring a musculoskeletal or joint-related injury increases.

Body weight distribution is another characteristic of pushup performance that can indicate the presence of compensational strategies. Most of the research in this area has focused on the changes in body weight force output between the up and down positions, or variations to the traditional push-up position. However, there has been a paucity of research investigating the changes to 
body weight distribution over the hands with the onset of fatigue when performing an extended bout of the traditional body weight push-ups $[17,18]$. Such information would be beneficial for identifying areas of muscular endurance weakness and those participants that may be at a greater risk for incurring musculoskeletal or joint-related injuries as they begin to tire. Therefore, the purpose of this study was two-fold. The first objective was to assess the muscle activity of the UT muscle throughout the entirety of a 2-minute standardized push-up bout to determine if the magnitudes of activation substantially change with the onset of temporary muscle fatigue. The second objective of this study was to assess the force output through the hands throughout the bout to investigate whether a change in body weight distribution centered through the hands may be related to the magnitude of activation in the UT muscle. It was hypothesized that the UT activity would increase with fatigue, while the percent of body weight over the hands would decrease, thereby lessening the load to be pushed.

\section{Materials and Methods}

Twenty-six uninjured college-aged males participated in this study (age $23.3 \pm 6.9$ years, height $1.76 \pm 0.9 \mathrm{~m}$, mass $84.2 \pm 11.3 \mathrm{~kg}$ ). All procedures were approved by the USMA Human Research Protection Program before commencing the study. Prior to participation, an informed written consent was obtained from each subject.

\section{Procedures}

Upon arrival to the single testing session, the age, height and weight were recorded for each subject. Subjects were then asked to position two individual force plates a comfortable distance apart under each hand. Wireless surface EMG electrodes were then placed bilaterally over the muscle belly of the upper trapezius (UT). A 5-second maximum voluntary isometric contraction (MVIC) was recorded for the UT by performing a bilateral isometric shoulder shrug with $85-120 \mathrm{~kg}$ dumbbells. Participants were given 5 minutes to warm-up and prepare on their own. Participants could assume the starting position (i.e. plank position with elbows fully extended) when ready prior to the start of the test. A running clock was visible to participants throughout the test to view the time remaining; no additional feedback was provided to participants during data collection.

\section{Data Analysis}

Raw EMG signals were full-wave rectified using the root mean square. From the smoothed data, the peak magnitudes were averaged for the $2^{\text {nd }}, 3^{\text {rd }}$ and $4^{\text {th }}$ repetition and the $4^{\text {th }}, 3^{\text {rd }}$ and $2^{\text {nd }}$ to last repetition of the 2-minute bout. These values were then normalized as a percentage of the peak root mean square of the 5 -second MVIC Force output data from the 2 force plates were plotted onto a forcetime graph. Like the EMG data, the peak force for each hand was averaged over the $2^{\text {nd }}, 3^{\text {rd }}$ and $4^{\text {th }}$ repetition and the $4^{\text {th }}, 3^{\text {rd }}$ and $2^{\text {nd }}$ to last repetition of the bout. These values were then normalized as a percentage of body weight for each participant.

\section{Statistical Analysis}

Data are presented as means \pm SD. Paired t-tests were used to determine if significant differences were present between the beginning and end of the exercise bout. Using Cohen's d effect sizes (ES) for all significant data, a value of 0.00 to 0.20 was a weak effect, 0.30 to 0.60 was considered to be a fair effect, 0.60 to 0.80 was considered to be a moderate effect and $\geq 0.80$ was considered to be a strong effect [19]. An alpha level of $p \leq 0.05$ was set for the level of significance for all statistical analyses. Sigma Stat 4.0 for Windows (Systat Software, Inc., San Jose, CA) was used to perform all statistical analyses.

\section{Results}

No statistical differences between right and left UT activity ( $p=0.24$ to 0.55 ). Therefore, the right and left EMG data were averaged together for the paired t-tests. No statistical difference was observed between the right and left hand at the start of the bout $(p=0.24)$. However, by the end of the bout significantly more force was produced through the right hand than that of the left $(p=0.04)$. Left and right force outputs were then added together and averaged for the start and end reps to give a representation of force output through the upper body throughout the bout. Significantly more force was produced through the hands at the start of the bout, when compared to the end $(\mathrm{p}=0.00)$. Normalized muscle activity and force outputs for the start and end of the bout are shown in Table 1. Both variables of interest decreased from the start of the bout to the end of the bout. However, the percent of body weight distributed over the hands (represented by the force output normalized to body weight) decreased significantly $(\mathrm{t}(22)=6.56, \mathrm{p}=0.00$, Cohen's $\mathrm{d}=1.67$ ), while the decrease in UT magnitude was marginal and not statistically significant $(\mathrm{t}(22)=1.23, \mathrm{p}=0.23$, Cohen's $\mathrm{d}=0.21)$.

Table 1: Normalized muscle activity and force outputs throughout the 2-minute continuous push-up bout.

\begin{tabular}{|c|c|c|c|}
\hline & Start & End & p-value \\
\hline UT EMG & $1.07(0.69)$ & $0.92(0.64)$ & 0.23 \\
\hline Force Output & $65.0(12.8)$ & $50.7(9.2)$ & $0.00^{*}$ \\
\hline
\end{tabular}

*Indicates significance at the 0.05 alpha level.

UT=Upper Trapezius; EMG = electromyography

UTEMG has been normalized to the maximum voluntary isometric contraction; Force Output has been normalized to body weight.

\section{Discussion}

The purpose of this study was to investigate if the UT muscle increases or decreases in activity as the participants begin to experience temporary muscle failure. The researchers' hypothesis that UT activity would increase with the onset of fatigue was not supported by the data. In fact, just the opposite occurred. While not significantly different from the beginning to the end of the bout, the magnitude of UT activity was greater at the beginning of the push- 
up bout than at the end. This indicates that as participants began to tire, they did not compensate by elevating their shoulders, instead they further depressed their shoulders while pressing up to the planking position. To the knowledge of the researchers of this study, no previous research has compared the UT activity under rested and fatigued conditions during the same exercise bout. However, limited UT activation levels are thought to be representative of an un-injured population, wherein an imbalance between the serratus anterior muscle group and UT have been associated with populations exhibiting shoulder pain, scapular winging and shoulder impingement none of which were reported by any of the participants in the present study $[20,21]$. Additionally, all participants were experienced with performing the traditional push-up prior to their participation in this study. Therefore, it is likely that they were aware of the optimal shoulder positioning for the exercise and were able to maintain it throughout the entirety of the bout.

The other variable of interest in the present study was the percentage of body weight positioned over the hands at the beginning and end of the 2-minute push-up bout. The researchers' hypothesis that the body weight through the hands would decrease as the participants began to tire was supported by the data. The initial percentage of body weight distributed over the hands $(65.0 \pm 12.8 \%)$ is consistent with that presented in previous literature $[17,22]$. While no research was found that presented such values at the end of a muscular endurance push-up bout, the values presented in this study from the end of the bout $(50.7 \pm 9.2 \%)$ are "normal" representations as the participants were well-practiced and familiar with the exercise and un-injured at the time of testing. Therefore, with a decrease in force output over the hands of $14.3 \%$ over the 2-minute bout, postural adjustments were made to lessen the load to be pushed by the upper body. This is likely a result of increased flexion at the hips (i.e. piking), which would distribute a greater percentage of body weight through the legs, although body angle measurements were not assessed in the present study. By decreasing the load over the upper body, the UT muscle would also not be required to activate to as great of an extent to perform the exercise, regardless of the level of temporary muscle fatigue being experience.

\section{Conclusion}

Muscle activity in the UT was not observed to differ significantly from the beginning to the end of 2-minute continuous push-up bout. In contrast, force output through the hands decreased significantly from the beginning to the end of the bout. This decrease in force output indicates that the percent of body weight being distributed over the hands was also decreased as the participants began to experience temporary muscle failure. To alleviate some of the load through the upper body, the participants likely incorporated a slight flexion at the hips, thereby distributing a greater load through the lower body. As a result, the UT were also not required to activate to as great of an extent at the end of the bout, as the load to be pushed had decreased. While additional research is needed in this area, it appears that UT activity is not increased as participants begin to tire if they are able to maintain a depressed scapular position throughout.

\section{References}

1. An KN, Chao EY, Morrey BF, Donkers MJ (1992) Intersegmental elbow joint load during the push-up. Biomed Sci Instrum 28: 69-74.

2. Cogley R, Archambault TA, Fibeger JF, Koverman MM, Youdas JW, et al. (2005) Comparison of muscle activation using various hand positions during the push-up exercise. J Strength Cond Res 19(3): 628-633.

3. Gouvali M, Boudolos K (2005) Dynamic and electromyographical analysis in variants of push-up exercise. J Strength Cond Res 19(1): 146151.

4. Lehman G, MacMillan B, MacIntyre I, Chivers M, Fluter M (2006) Shoulder muscle EMG activity during push-up variations on and off a swiss ball. Dyn Med 5(7): 1-7.

5. Chou P, Hsu H, Chou Y (2011) Effect of push-up speed on elbow joint loading. Journal of Medical and Biological Engineering 31(3): 161-168.

6. Marcolin G, Petrone N, Moro T, Battaglia G, Bianco A, et al. (2015) Selective activation of shoulder, trunk, and arm muscles: A comparative analysis of different push-up variants. J Athl Train 50(11): 1126-1132.

7. Kim Y, Kim DY, Ha MS (2016) Effect of the push-up exercise at different palmar width on muscle activities. J Phys Ther Sci 28(2): 446-449.

8. Sandhu J, Mahajan S, Shenoy S (2008) An electromyographic analysis of shoulder muscle activation during push-up variations on stable and labile surfaces. Int J Shoulder Surg 2(2): 30-35.

9. Anderson G, Geatz M, Matt H, Twist P (2013) Comparison of EMG activity during stable and unstable push-up protocols. European Journal of Sport Science 13(1): 42-48.

10. Beach T, Howarth SJ, Callaghan JP (2008) Muscular contribution to lowback loading and stiffness during standard and suspended push-ups. Hum Mov Sci 27(3): 457-472.

11. Borreani S, Calatayud J, Colado JC, Tella V, Moya-Nájera D, et al. (2015) Shoulder muscle activation during stable and suspended push-ups at different heights in healthy subjects. Phys Ther Sport 16(3): 248-254.

12. Vossen J, Kramer J, Burke D, Vossen D (2000) Comparison of dynamic push-up training and plyometric push-up training on upper-body power and strength. Journal of Strength and Conditioning Research 14(3): 248253.

13. Garcia-Masso X, Colado JC, González LM, Salvá P, Alves J, et al. (2011) Myoelectric activation and kinetics of different plyometric push-up exercises. Journal of Strength and Conditioning Research 25(7): 20402047.

14. Herrington L, Waterman R, Smith L (2015) Electromyographic analysis of shoulder muscles during press-up variations and progressions. J Electromyogr Kinesiol 25(1): 100-106.

15. Martins J, Tucci HT, Andrade R, Araújo RC, Bevilaqua-Grossi D, et al. (2008) Electromyographic amplitude ratio of serratus anterior and upper trapezius muscles during modified push-ups and bench press exercises. J Strength Cond Res 22(2): 477-484.

16. Contreras B, Schoenfeld B, Mike J, Tiryaki-Sonmez G, Cronin J, et al (2012) The biomechanics of the push-up. Strength Cond J 34(5): 41-45.

17. Ebben W, Wurm B, Vander Zanden TL, Spadavecchia ML, Durocher JJ, et al. (2011) Kinetic analysis of several variations of push-ups. J Strength Cond Res 25(10): 2891-2894

18. Suprak D, Dawes J, Stephenson MD (2011) The effect of position on the percentage of body mass supported during traditional and modified push-up variants. J Strength Cond Res 25(2): 497-503. 
19. Urdan $T$ (2007) Statistical significance, effect size, and confidence intervals. Statistics in Plain English. New York, NY, USA, pp.73-92.

20. Ludewig P, Hoff MS, Osowski EE, Meschke SA, Rundquist PJ (2004) Relative balance of serratus anterior and upper trapezius muscle activity during push-up exercises. Am J Sports Med 32(2): 484-493.
21. Suprak D, Bohannon J, Morales G, Stroschein J, San Juan JG (2013) Scapular kinematics and shoulder elevation in a traditional push-up. J Athl Train 48(6): 826-835.

22. Mier C (2014) Differences between men and women in percentage of body weight supported during the push-up exercise. International Journal of Exercise Science 7(2): 161-168.

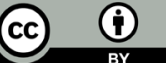

Creative Commons Attribution 4.0 International License

For possible submissions Click Here

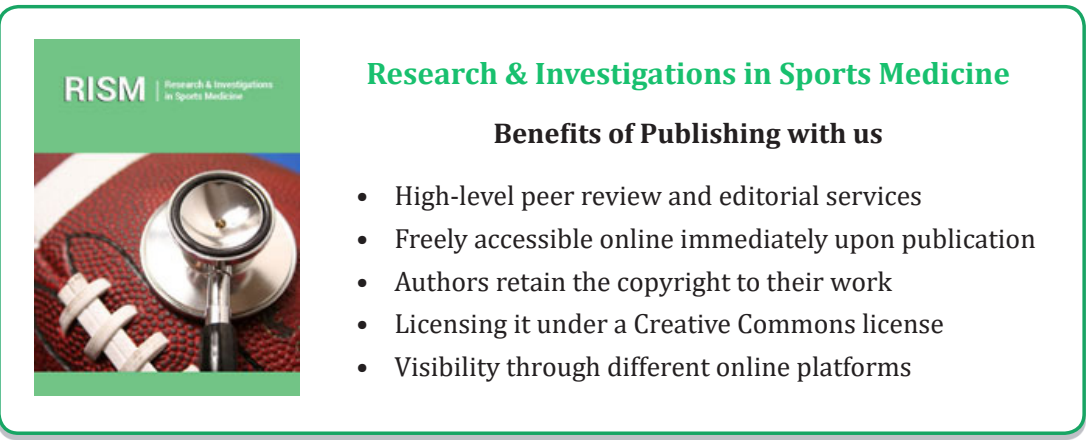

